(C) Dereito Vol.29, n02:83-96 (Xullo-Decembro, 2020) • ISSN 1132-9947

\title{
CULTURA E DIRITTO AMBIENTALE
}

Cultura y derecho ambiental

Culture and environmental law

DOI: http://dx.doi.org/10.15304/dereito.29.2.6898

Livio Perra ${ }^{1}$

Professore a Contratto di Legislazione dei Beni Culturali

Università degli Studi di Sassari

Iperra@uniss.it

\section{Riassunto}

Nel presente articolo l'autore pone l'accento sullo stretto legame che intercorre tra il diritto e la cultura. Il mondo del diritto allo stesso tempo trova spunti di riflessione nella tradizione e diventa manifestazione della cultura. In materia di diritto ambientale questa connessione trova il proprio apice nei diritti bioculturali e nei diritti delle entità naturali non umane. Nei nuovi sistemi di protezione ambientale poggiati sulle solide basi della cultura, l'autore indaga anche il nuovo ruolo dell'essere umano. Lo scopo del presente lavoro consiste nel dimostrare come, partendo dalla cultura, si possa giungere all'innovazione giuridica. L'esempio offerto dai nuovi sistemi di protezione ambientale, che si pongono come soluzioni alle problematiche ambientali, muove la riflessione dell'autore nell'intero lavoro ed egli giunge alla conclusione che l'ordinamento giuridico possa trovare soluzioni utili per risolvere i problemi del presente traendo ispirazione dal sapere antico e dalla cultura dei popoli che compongono $i$ Paesi.

Parole chiave: ritto; cultura; diritti bioculturali; diritti della natura.

\begin{abstract}
In this article, the author emphasizes the close link between law and culture. The world of law at the same time finds food for thought in tradition and becomes a manifestation of culture. In the field of environmental law, that connection finds its apex in the biocultural rights and in the rights of non-human natural entities. In the new environmental protection systems based on the solid foundations of culture, the author also investigates the new role of the human being. The purpose of this work is to demonstrate how, starting from culture, legal innovation can be achieved. The example offered by the new environmental protection systems, which act as solutions to environmental problems, moves the author's reflection throughout the work and he comes to the conclusion that the legal system can find useful solutions to solve the problems of the present, drawing inspiration from ancient knowledge and from the culture of the peoples that make up the Countries.
\end{abstract}

${ }^{1}$ https://orcid.org/0000-0002-1045-1206

Recibido: 20/09/2020. Aceptado: 28/12/2020. 
Keywords: law; culture; biocultural rights; rights of nature.

\section{Resumen}

En este artículo, el autor enfatiza el estrecho vínculo entre el derecho y la cultura. Al mismo tiempo, el mundo del derecho encuentra alimento para el pensamiento en la tradición y se convierte en una manifestación de la cultura. Con respecto al derecho ambiental, esta conexión encuentra su ápice en los derechos bioculturales y en los derechos de las entidades naturales no humanas. En los nuevos sistemas de protección ambiental basados en los sólidos cimientos de la cultura, el autor también investiga el nuevo papel del ser humano. El propósito de este trabajo es demostrar cómo, a partir de la cultura, se puede lograr la innovación legal. El ejemplo ofrecido por los nuevos sistemas de protección ambiental, que actúan como soluciones a los problemas ambientales, mueve la reflexión del autor a lo largo del trabajo y él llega a la conclusión de que el sistema legal puede encontrar soluciones útiles para resolver los problemas del presente, inspirándose en el conocimiento antiguo y en la cultura de los pueblos que componen los Países.

Palabras clave: derecho; cultura; derechos bioculturales; derechos de la naturaleza.

\section{INTRODUZIONE}

Nello scenario odierno, dove molti Paesi sono alla ricerca di sempre più efficaci soluzioni alla problematica ambientale, alcuni di essi trovano un punto di partenza su cui poggiare i propri sistemi di protezione ambientale sui valori dei popoli che compongono il loro territorio. Lo stretto rapporto tra diritto e cultura mostra tutta la sua forza nei sistemi di protezione ambientale incentrati sui diritti bioculturali e sui diritti della natura o delle sue entità. I valori culturali delle comunità etniche e dei popoli indigeni costituiscono fonte d'ispirazione per la costruzione dei nuovi sistemi di protezione ambientale che pongono al proprio centro i diritti bioculturali o i diritti della natura e delle sue entità. In particolare, il fenomeno osservabile è denominato etnosviluppo giuridico. Si tratta, cioè, dell'utilizzo di tutto il bagaglio della tradizione dei popoli che abitano i territori per innovare il diritto e costruire il futuro alla luce delle aspirazioni e dei desideri del popolo.

Nel presente lavoro, sono analizzati i rapporti tra diritto e cultura che intercorrono nel diritto ambientale. A tal fine, nel $\S 2$., l'attenzione è riposta sullo stretto legame tra diritto e cultura. In particolare, si osserva come in materia ambientale i valori della tradizione sono fatti propri dal diritto. Il diritto non solo trae ispirazione dalla cultura per innovare e costruire nuovi sistemi di protezione ambientale, ma diviene allo stesso tempo il veicolo con cui la medesima cultura si manifesta e diviene elemento caratterizzante nelle soluzioni escogitate per rispondere alla problematica ambientale.

Nel § 3., è analizzato il fenomeno dell'etnosviluppo giuridico. Si osserva come alcuni Paesi hanno utilizzato il sapere antico ed i propri valori culturali per costruire un sistema di protezione ambientale che risponda ai 
loro desideri e alle loro aspirazioni. La natura e le sue entità ottengono nel mondo giuridico la considerazione che rivestono nella cultura dei popoli che compongono gli Stati. Il diritto si innova e si evolve, utilizza i valori culturali per poggiare le fondamenta dei nuovi sistemi di protezione ambientale.

Il $\S 4$., è dedicato ai diritti bioculturali. In particolare, viene delineata dapprima una definizione di questa categoria emergente di diritti. In seguito, ripercorrendo anche alcune ricostruzioni giurisprudenziali, è ricostruito il quadro teorico che sta alla base dei diritti bioculturali. Infine, è sottolineato come tali diritti abbiano come fondamento e tutelino le forme di gestione e di amministrazione dei territori della tradizione.

Nel $\S 5$., sono esaminati i diritti delle entità naturali non umane. Questi diritti vengono chiamati diritti ecologici e si differenziano dai classici diritti ambientali, in quanto sono diritti attribuiti alla natura o alle sue entità in quanto tali, cioè la titolarità è in capo alla natura o alle sue entità. Affiora, poi, una ricostruzione giurisprudenziale secondo cui tale nuova categoria di diritti ha trovato la strada per emergere nel cammino tracciato dai diritti bioculturali, costituendo una ulteriore evoluzione nel pensiero giuridico.

Nel $\S 6$. , è indagato il ruolo dell'essere umano nei nuovi sistemi di protezione ambientale. In particolare, si rileva il delinearsi di una nuova concezione di essere umano. L'uomo è ora colui il quale presta attenzione alle proprie azioni in un'ottica di ecosostenibilità e può contribuire alla difesa della natura grazie ai meccanismi di partecipazione. È sottolineata l'importanza dell'informazione, la quale si pone come strumento utile per rendere possibili le forme partecipative nei procedimenti dove si assumono le decisioni in materia ambientale.

Nel trarre le conclusioni dell'analisi effettuata e delle riflessioni formulate, viene posto l'accento sullo stretto legame tra diritto e cultura. Questo legame ha influenzato fortemente il mondo giuridico che ha costruito nuovi sistemi di protezione ambientale dove sono rispecchiati i valori culturali dei popoli che abitano i territori.

\section{LO STRETTO LEGAME TRA DIRITTO E CULTURA}

I nuovi sistemi di protezione ambientale che predicano i diritti delle entità naturali non umane hanno in comune il fatto che si poggiano sulle solide basi della cultura e della tradizione dei popoli. In Ecuador e Bolivia viene trasfusa nei testi giuridici la concezione indigena di Pachamama, la Madre Terra che riveste un valore sacrale nella cultura dei popoli che compongono rispettivamente $\mathrm{i}$ due Stati ${ }^{2}$. In Nuova Zelanda, il fiume

\footnotetext{
${ }^{2}$ Nelle Costituzioni di Ecuador e Bolivia e, più in generale, nei testi normativi in materia ambientale di entrambi i Paesi emerge spesso l'utilizzo di termini nelle lingue indigene. Ad esempio nel Preambolo della Costituzione dell'Ecuador, il concetto di natura di matrice occidentale è affiancato a quello di Madre Terra [Pachamama] della cultura dei popoli indigeni. Si veda in tal senso: L. PERRA, "Tradition Can Save the Future of Nature: Biocentric View of Law", in E.G. DOBBINS, M.L. PIGA, L. MANCA, (coords.), Environment, Social Justice, and the Media in the Age of the Anthropocene, Lexington Books, Lanham, MD, 2020, p. 341: «from the first lines of Ecuador's constitution, as highlighted by many commentators and academics, the western concept of Nature is likened to indigenous
} 
Whanganui River [Te Awa Tupua] ha due antenati in comune con il popolo Whanganui Iwi e da questa credenza nasce il forte desiderio degli individui di prendersi cura del medesimo fiume ${ }^{3}$.

La portata dei valori culturali in materia ambientale in tali Paesi si può comprendere se si sposa una concezione del diritto che tenga in conto lo sfondo culturale, il contesto in cui i testi giuridici sono inseriti e i desideri e le aspirazioni per il futuro dei popoli.

Seguendo l'indicazione di metodo offerta da Peter Häberle, lo sguardo non può essere sottratto a quel continuo intreccio che è presente tra i valori della cultura dei popoli ed i testi giuridici.

Häberle ${ }^{4}$, in riferimento alle Costituzioni, parla di due livelli strettamente interconnessi osservabili in tali testi. Lo spirito delle Costituzioni [Geist der Verfassungen] è formato dallo spirito delle costituzioni generale, che determina il tipo o il modello di Stato costituzionale, e dallo spirito dei singoli popoli, che vive nelle Costituzioni e ne forma il substrato ${ }^{5}$.

Questo discorso caratterizza non solo le Costituzioni, ma è osservabile anche nelle leggi e negli altri atti che formano i nuovi ed articolati sistemi di protezione ambientale. Infatti, in materia ambientale in Ecuador, in Bolivia e in Nuova Zelanda, si osserva l'emergere dirompente della cultura dei popoli che compongono tali Paesi ed essa si esplica in diverse forme.

Per prima cosa va osservata la scelta linguistica. Accanto alle lingue europee, sono presenti termini delle lingue indigene. Sono termini densi di

people's concept of Mother Earth (Pachamama)». Si veda, ad esempio, a tal proposito: E. GUDYNAS, "Desarrollo, derechos de la naturaleza y Buen vivir despues de Montecristi," in G. WEBER (coord.), Debates sobre cooperación y modelos de desarrollo. Perspectivas desde la sociedad civil en el Ecuador, Centro de Investigaciones CIUDAD y Observatorio de la Cooperación al Desarrollo, Quito, 2011, p. 87. Si tratta non solo di un affiancamento terminologico, ma i termini permeati dalla cultura dei popoli indigeni portano con sé il proprio significato. Ad esempio nel Preambolo della Costituzione della Bolivia, la Pachamama è colei che contribuisce a rifondare il Paese, effondendo la sua forza. Tale Preambolo afferma: «cumpliendo el mandato de nuestros pueblos, con la fortaleza de nuestra Pachamama y gracias a Dios, refundamos Bolivia».

${ }^{3}$ Si veda a tal proposito: WHANGANUI IWI AND THE CROWN, Tūtohu Whakatupua, 30 August 2012, paragrafo 1.3.: «the desire of Whanganui Iwi to care, protect, manage and use the Whanganui River through the kawa and tikanga maintained by the descendants of Ruatipua and Paerangi». Si veda anche: E.C. HSIAO, "Whanganui River Agreement Indigenous Rights and Rights of Nature", Environmental Policy and Law, v. 42, n.6, 2012, p. 371.

${ }^{4}$ Si veda a tal proposito: P. HÄBERLE, Verfassungslehre als Kulturwissenschaft, Duncker \& Humblot, Berlin, 1998, p. 29.

5 Si veda a tal proposito: ibid.: "Jede Verfassungslehre muß etwas vom "Geist der Verfassungen" einzufangen suchen - sie hätte sich an Montesquieus' "Geist der Gesetze" zu orientieren, wäre dieser Anspruch nicht zu unbescheiden. Etwas vom "Geist der Verfassungen" wird jedoch in den Verfassungstexten greifbar, vor allem in ihrer kontemporär und geschichtlich begriffenen "Entwicklung" als Textstufen-Vorgang. Dieser "Geist" wirkt auch in den kulturwissenschaftlich zu erschließenden kulturellen KonTexten. Dabei ist zwischen zwei Ebenen zu unterscheiden: es gibt einen allgemeinen, typusorientiert zu erarbeitenden "Geist der Verfassungen" (des Verfassungsstaates) und es gibt einen sehr individuell geprägten "Geist der einzelnen Völker", die in, nach und "unter" Verfassungen leben». 
un forte significato simbolico, termini spesso non perfettamente traducibili per via del carico concettuale che portano con sé.

In seconda battuta, si devono evidenziare i molteplici richiami concettuali. Vi sono chiari riferimenti alla cosmovisione dei popoli indigeni. Si tratta, cioè, di termini che trasportano all'interno dei testi giuridici stili, modi di vivere $e$, più in generale, la stessa concezione che gli individui hanno di loro stessi ${ }^{6}$. L'essere umano è inserito in un contesto natura più ampio, dove non è l'uomo a primeggiare, ma quest'ultimo contribuisce ad una armonia globale con tutti gli altri esseri con cui condivide gli spazi della propria esistenza.

In tal modo, utilizzando le parole di Jean Cruet $^{7}$, il diritto esprime la società. I testi giuridici non solo ordinano la vita della società prescrivendo condotte o vietando azioni, ma diventano il veicolo con cui la cultura si manifesta. I valori, così veicolati, contribuiscono ad affermare l'identità dei popoli, la loro storia, la loro tradizione, i loro desideri e le speranze per il futuro.

\section{ETNOSVILUPPO GIURIDICO}

La nostra epoca è caratterizzata da un consumismo sfrenato, prevale la ricerca del guadagno economico e lo sfruttamento delle risorse oltre ogni ragionevole limite. Questo modello che è stato spesso identificato con il progresso ed è apparso per anni come il modo in cui migliorare le

\footnotetext{
${ }^{6}$ L'esempio maggiormente calzante del fenomeno è costituito dall'ingresso del concetto del Buen vivir [in lingua Quechua: sumak kawsay] nei testi giuridici dell'Ecuador e del Vivir Bien [in lingua Aymara: suma qamaña] in quelli della Bolivia. Il Buen Vivir è posto nella Costituzione dell'Ecuador come un obiettivo da raggiungere. La Costituzione afferma che il popolo dell'Ecuador decide di costruire «una nuova forma di convivenza civile, nella diversità e in armonia con la natura, per conseguire il buen vivir, il sumak kawsay [una nueva forma de convivencia ciudadana, en diversidad y armonía con la naturaleza, para alcanzar el buen vivir, el sumak kawsay]». La Costituzione della Bolivia prevede che il valore del Vivir Bien orienterà la politica pubblica dello Stato. Cfr. L. PERRA, "Etnodesarrollo jurídico y protección del medio ambiente", Veredas do Direito: Direito Ambiental e Desenvolvimento Sustentável, v. 16, n. 34, 2019, p. 74. Si veda a tal proposito: F. HUANACUNI MAMANI, Buen Vivir / Vivir Bien. Filosofía, políticas, estrategias y experiencias regionales andinas, Coordinadora Andina de Organizaciones Indígenas CAOI, Lima, 2010, p. 18. Il Buen Vivir e il Vivir Bien sono concetti che derivano dalla cosmovisione indigena. Essi caratterizzano il modo di vivere e di percepire il mondo. Questi valori sono presenti nella cultura dei popoli indigeni che abitano nel Continente americano. Ciascuna di queste culture ha le proprie peculiarità, che derivano dalla loro stessa identità, ma vi è un simile modo di concepire la vita in una dimensione di armonia ed equilibrio con il mondo circostante. Si veda a tal proposito: F. HUANACUNI MAMANI, op. cit., p. 24: «en gran parte de los pueblos de la región andina de Colombia, Ecuador, Bolivia, Perú, Chile y Argentina, y en los pueblos ancestrales (primeras Naciones) de Norteamérica pervive la Cosmovisión Ancestral o Visión Cósmica, que es una forma de comprender, de percibir el mundo y expresarse en las relaciones de vida. Existen muchas naciones y culturas en el Abya Yala, cada una de ellas con sus propias identidades, pero con una esencia común: el paradigma comunitario basado en la vida en armonía y el equilibrio con el entorno».

7 Si veda a tal proposito: J. CRUET, La vie du droit et l'impuissance des lois, Ernest Flammarion, Paris, 1908, p. 336: «Le droit ne domine pas la société, il l'exprime». Si veda anche: F. VIOLA, "Il futuro del diritto", Persona y Derecho, n. 79, 2018, p. 10.
} 
condizioni di vita umane si trova oggi in crisi. Il Pianeta è stato messo a dura prova dalla attività poco coscienziosa degli individui. La natura vista come una inesauribile fonte di risorse sta suonando continui campanelli d'allarme e l'attenzione globale ha gradatamente compreso che la salute della Terra, o meglio, la scarsa attenzione ad essa può condurre la razza umana verso una deriva non piacevole. In tal modo risuonerebbe alquanto familiare il proverbio pronunciato dal popolo Cree, secondo il quale: «solo quando l'ultimo albero sarà morto e l'ultimo fiume sarà avvelenato, e I'ultimo pesce sarà catturato, realizzeremo che non possiamo mangiare il denaro»8.

Per scongiurare una simile deriva ambientale, molti Paesi ritengono che sia utile abbandonare gli schemi dettati da comportamenti e stili di vita poco virtuosi che rischiano addirittura di accelerare il processo che potrebbe portare alla rovina del Pianeta. Numerose sono le politiche pubbliche tese all'interesse generale della preservazione della natura, dei suoi ritmi e cicli vitali. Alcuni Stati hanno cercato nella tradizione dei popoli che abitano i propri territori la chiave per risollevare le sorti del nostro mondo e avere chiare indicazioni di metodo riguardo ai comportamenti da adottare e su di questi valori riscoperti hanno poggiato le basi per innovare giuridicamente, addivenendo ad evoluti sistemi di protezione ambientale.

Nello specifico si è trattato di una vera e propria riscoperta dei valori della tradizione e della sapienza degli antenati. Il passato in questo modo diviene la chiave per il presente e per salvare il futuro.

Nelle costruzioni giuridiche che hanno scelto come soluzione alla problematica ambientale l'affermazione dei diritti della natura o delle sue entità o l'introduzione dei diritti bioculturali è osservabile quel fenomeno denominato etnosviluppo giuridico. Con il termine etnosviluppo si fa riferimento alla definizione delineata da Guillermo Bonfil Batalla, secondo il quale: «per etnosviluppo si intende l'esercizio della capacità sociale di un popolo per costruire il suo futuro, utilizzando per questo gli insegnamenti della sua esperienza storica e le risorse potenziali della propria cultura, in accordo con un progetto definito secondo i propri valori e aspirazioni»9 . Questo metodo ha fatto ingresso nel mondo giuridico e vi sono molteplici esempi adducibili, si pensi ad esempio al nuovo costituzionalismo andino dell'ultimo decennio ed alle numerose novità in materia ambientale ${ }^{10}$, agli

$8 \mathrm{Si}$ veda a tal proposito: C. MASER, Earth in our care: ecology, economy, and sustainability, Rutgers University Press, New Brunswick, N.J., 2009, p. 142: «Only when the last tree has died, and the last river has been poisoned, and the last fish been caught will we realize we cannot eat money».

9 G. BONFIL BATALLA, "El etnodesarrollo: sus premisas jurídicas, políticas y de organización", in G. BONFIL BATALLA, M. IBARRA, S. VARESE, D. VERISSIMO, J. TUMIRI, et al. (F. Rojas Aravena, coord.), América Latina: Etnodesarrollo y Etnocidio, Ediciones FLACSO, San José, 1982, p. 133: «por etnodesarrollo se entiende el ejercicio de la capacidad social de un pueblo para construir su futuro, aprovechando para ello las enseñanzas de su experiencia histórica y los recursos reales y potenciales de su cultura, de acuerdo con un proyecto que se defina según sus propios valores y aspiraciones».

${ }^{10}$ In Ecuador e in Bolivia si osserva, in materia ambientale, il riconoscimento della natura come un soggetto con veri e propri diritti. La natura non è più solamente un oggetto 
accordi tra il popolo Iwi ed il Governo neozelandese che hanno portato agli atti legislativi che hanno riconosciuto il fiume Whanganui River [Te Awa Tupua] come legal person con veri e propri diritti.

Per comprendere quanto abbia contribuito la cultura nelle nuove prospettive giuridiche volte alla difesa ambientale, è utile considerare quali siano i valori della tradizione ai quali si è fatto richiamo esplicito o implicito nelle nuove costruzioni giuridiche.

Un punto in comune che hanno le prime civiltà apparse sul Pianeta è il forte sentimento che le lega all'ambiente e al proprio territorio. In tal senso, Fernando Huanacuni Mamani ${ }^{11}$ rileva come tutte le culture hanno un proprio modo di vedere il mondo. La concezione che essi ne hanno è denominata cosmovisione. Nella visione del mondo degli antenati, tutto è connesso e la vita è incentrata all'armonia e all'equilibrio con la natura. Nei popoli andini e nordamericani sopravvive la cosmovisione ancestrale nel modo di percepire e relazionarsi al mondo. Il paradigma comunitario risulta fondato sull'armonia e sull'equilibrio con il proprio ambiente.

\section{I DIRITTI BIOCULTURALI}

Al fine di comprendere in cosa consistano i diritti bioculturali, occorre soffermare l'attenzione dapprima sulla definizione. Tale definizione è individuata da Gianfrancesco Zanetti ${ }^{12}$. I diritti bioculturali sono un gruppo di diritti umani collettivi fondati sul ruolo di stewardship. La gestione o amministrazione dell'ambiente si esplica nel ruolo che ancor oggi esercitano le comunità locali ed i gruppi indigeni sull'ambiente. Si tratta di diritti poggiati su delle pratiche che variano in base al contesto e alla cosmovisione della comunità. Il punto in comune di queste pratiche su cui si innalzano tali diritti è il fine della conservazione dell'ambiente.

I diritti bioculturali collegano a doppio filo la cultura dei popoli alla natura. Nello specifico sono diritti tesi alla conservazione dei modi tradizionali delle comunità locali di rapportarsi alla natura, che si traducono nella conservazione di un aspetto fondamentale della identità del gruppo.

La categoria dei diritti bioculturali trova un'approfondita analisi nella sentenza T-622/16 della Corte Costituzionale colombiana.

In tale sentenza, la Corte individua dapprima la definizione di diritti bioculturali. I diritti bioculturali sono i diritti delle comunità etniche di amministrare e tutelare in maniera autonoma i propri territori e le risorse

\footnotetext{
asservito all'essere umano, ma è la Madre Terra. Essa nella cultura dei popoli indigeni che compongono lo Stato è personificata e riveste un valore spesso sacrale. L'uomo concorre con le altre entità naturali a formare un sistema che si regge sui reciproci equilibri. Il benessere generale dipende da quello delle singole parti. La vera novità in campo giuridico è, pertanto, costituita dall'allontanamento dall'antropocentrismo. Il diritto non è più costruito solo in funzione dell'essere umano, ma all'interno dei due ordinamenti giuridici vi sono altre entità nobilitate al rango di soggetto.

${ }^{11} \mathrm{Si}$ veda a tal proposito: F. HUANACUNI MAMANI, op. cit., p. 24.

12 G. ZANETTI, "Diritti bioculturali: percorrendo la strada che separa diverse tradizioni", Diritto \& Questioni Pubbliche, v. 19, n. 1, 2019, p. 269.
} 
naturali. Questa amministrazione e tutela segue le leggi, le consuetudini di tali comunità ${ }^{13}$.

Vi è un collegamento molto stretto tra natura e cultura. La biodiversità e I'insieme di tradizioni, usi, costumi culturali e spirituali dei popoli sono inscindibili ed interdipendenti.

Gli stili di vita delle comunità etniche sono basati sul territorio, con il quale esse hanno un particolare legame culturale e spirituale.

Con il termine comunità la stessa Corte sottolinea che deve intendersi un gruppo di persone il cui modo di vivere è determinato dal suo ecosistema ${ }^{14}$.

I diritti bioculturali tutelano un modo di vivere che al suo interno contiene la conservazione e l'uso sostenibile dell'ecosistema. Lo stretto legame tra la comunità e l'ecosistema è tale da collegare la conservazione e l'uso sostenibile delle risorse naturali alla amministrazione e gestione del territorio ed ai diritti alla cultura, costituiti dal bagaglio di conoscenze e pratiche delle comunità etniche.

Al fine di proteggere e conservare lo stile di vita delle comunità etniche occorre proteggere la biodiversità e, più in generale il territorio, perché costituiscono le condizioni necessarie alla sopravvivenza dei modi di essere, di percepire e conoscere il mondo propri delle medesime comunità ${ }^{15}$.

Secondo tale lettura, i diritti bioculturali assurgono a presidio dei modi di vivere delle comunità etniche. Queste comunità hanno uno stretto legame con la natura. Nella loro cultura essi sono parte di un sistema natura dove vi è una forte connessione con le altre entità naturali. Proteggere il territorio è, dunque, indispensabile per la stessa conservazione della loro cultura, della loro identità. L'amministrazione e gestione del proprio territorio diviene di conseguenza espressione della propria cultura, della stessa identità e si esplica nel rispetto dell'insieme delle leggi, delle consuetudini, degli usi, dei costumi e delle pratiche rituali dello stesso popolo. Negare l'amministrazione del territorio secondo le forme tradizionali equivarrebbe a svilire la cultura, la tradizione e l'identità delle comunità etniche.

\section{I DIRITTI DELLE ENTITÀ NATURALI NON UMANE}

I diritti delle entità naturali non umane sono quei diritti attribuiti alla natura o a particolari entità naturali non umane riconosciute dagli ordinamenti giuridici di alcuni Paesi come soggetti o persone.

Essi vengono distinti dai diritti umani. L'essere umano entra in gioco soltanto con un ruolo di rappresentanza o tutela (in base all'ordinamento giuridico che prevede tali forme) per l'esercizio dei meccanismi posti a tutela dei diritti delle entità naturali non umane. Quando la natura o le sue entità non possono provvedere alla cura dei propri interessi, saranno gli

\footnotetext{
${ }^{13}$ CORTE CONSTITUCIONAL DE COLOMBIA, Sentencia T-622/16, 2016, punto 5.11.

${ }^{14}$ CORTE CONSTITUCIONAL DE COLOMBIA, Sentencia T-622/16, 2016, punto 5.14 .

${ }^{15}$ CORTE CONSTITUCIONAL DE COLOMBIA, Sentencia T-622/16, 2016, punto 5.18 .
} 
esseri umani ad esercitare le azioni dinnanzi ai competenti tribunali o proporre le istanze presso le amministrazioni pubbliche.

La netta distinzione tra i diritti della natura ed i diritti umani è osservabile in Ecuador, come evidenziato da Alberto Acosta.

Acosta $^{16}$ sottolinea la presenza nella Costituzione dell'Ecuador di due categorie distinte di diritti: i diritti ambientali ed i diritti ecologici.

I diritti ambientali sono osservabili nell'articolo 14 della Costituzione dell'Ecuador e fanno riferimento ai diritti degli esseri umani a vivere in un ambiente sano.

Del tutto autonomi e indipendenti dagli interessi degli esseri umani vi sono i diritti della natura, denominati diritti ecologici. Questi diritti sono appannaggio della sola natura, sono, cioè, diritti di cui la natura è titolare di per se stessa. In particolare, essi proteggono i cicli vitali, i processi evolutivi, le specie e le aree naturali.

Una teoria espressa dalla Corte Costituzionale colombiana vede nella nuova categoria dei diritti delle entità naturali non umane uno sviluppo del nuovo approccio giuridico costituito dai diritti bioculturali. A tal fine, è utile ripercorrere alcuni passaggi concettuali contenuti nella sentenza T-622/16 della Corte Costituzionale colombiana. Questi diritti, secondo la ricostruzione operata dalla medesima Corte, hanno trovato una porta aperta sulla scia offerta dal sistema dei diritti bioculturali.

La Corte afferma che nel «nuovo approccio giuridico denominato diritti bioculturali, la cui premessa centrale è la relazione di profonda unità e interdipendenza tra natura e specie umana e che ha come conseguenza una nuova comprensione socio-giuridica in cui la natura e il suo ambiente devono essere presi sul serio e con pienezza dei diritti. Cioè, come soggetti di diritti»17. In quest'ottica la «giustizia con la natura» deve superare lo scenario umano e consentire che la natura possa divenire soggetto di diritti ${ }^{18}$.

Nel prevedere i diritti della natura o delle sue entità, il mondo giuridico ha recepito la visione delle comunità etniche e dei popoli indigeni, secondo cui la natura o le sue entità sono esseri viventi che al pari dell'essere umano compongono un sistema di vita più ampio. Viene riconosciuto quel valore sacrale della natura e delle sue componenti, che è presente nella cultura delle comunità etniche che compongono un determinato Stato. Essere considerati soggetti alla pari in un sistema giuridico comporta che siano attribuiti i medesimi gradi di tutela e, nel caso specifico, ciò si sostanzia nell'affermazione dei diritti delle entità naturali non umane.

\footnotetext{
${ }^{16}$ Si veda a tal proposito: A. ACOSTA, "Los Derechos de la Naturaleza. Una lectura sobre el derecho a la existencia", in A. ACOSTA, E. MARTÍNEZ (coord.), La naturaleza con derechos. De la filosofía a la política, Abya-Yala, Quito, 2011, pp. 354-355.

17 CORTE CONSTITUCIONAL DE COLOMBIA, Sentencia T-622/16, 2016, punto 9.28.: «nuevo enfoque jurídico denominado derechos bioculturales, cuya premisa central es la relación de profunda unidad e interdependencia entre naturaleza y especie humana, y que tiene como consecuencia un nuevo entendimiento socio-jurídico en el que la naturaleza y su entorno deben ser tomados en serio y con plenitud de derechos. Esto es, como sujetos de derechos».

${ }^{18} \mathrm{Cfr}$. CORTE CONSTITUCIONAL DE COLOMBIA, Sentencia T-622/16, 2016, punto 9.31.
} 


\section{L'ESSERE UMANO}

Di fronte ai nuovi sistemi di protezione ambientale, non può essere trascurato l'aspetto che riguarda la relazione intercorrente tra gli individui e tali sistemi.

Per prima cosa è necessario effettuare qualche considerazione concernente gli stili di vita delle persone.

Dinnanzi ai diritti della natura o delle sue entità si possono porre varie questioni sull'incidenza sull'attività umana. In particolare, molti comportamenti del tutto legittimi o tollerati prima della loro introduzione, potrebbero entrare in contrasto con le nuove previsioni ${ }^{19}$. A tal fine deve essere considerato il motivo per cui questa costruzione giuridica è venuta in essere. Questi sistemi nascono per contrastare l'eccessivo e sconsiderato utilizzo delle risorse naturali o le modalità che possono cagionare un pregiudizio alle entità naturali e non certo per impedire le attività umane tese al procacciamento di cibo ${ }^{20}$. La caccia, la pesca e I'utilizzo dei terreni per l'agricoltura non è minacciata da tale previsione. Anche l'attività mineraria non vede un'assoluta esclusione. Le limitazioni ed i divieti concernono tutte quelle attività che possono arrecare un danno o un pericolo all'ambiente. In un'ottica di preservazione dell'ambiente ogni attività deve essere pensata ecosostenibilmente. Ad esempio, le sostanze nocive non potranno essere utilizzate per porre in essere l'attività mineraria o nell'agricoltura e nell'allevamento. L'utilizzo di sostanze inquinanti per i procedimenti estrattivi o l'utilizzo di pesticidi che possano arrecare un pregiudizio all'ambiente circostante devono essere scoraggiati ${ }^{21}$. Altro aspetto che merita di essere considerato è la volontà di limitare gli sprechi. Con un sistema che prevede i diritti delle entità naturali non umane è più facile per l'ordinamento giuridico reagire alle cattive condotte ed evitare i possibili danni ambientali, perché una violazione dei diritti di queste entità trova da subito in risposta i meccanismi adatti.

19 Si veda a tal proposito: L. PERRA, "Etnodesarrollo jurídico y protección del medio ambiente", Veredas do Direito: Direito Ambiental e Desenvolvimento Sustentável, v. 16, n. 34, 2019, p. 76. Si vedano inoltre: E.R. ZAFFARONI, "La naturaleza como persona: de la Pachamama a la Gaia", in C. ESPINOSA GALLEGOS-ANDA, C. PÉREZ FERNÁNDEZ, (coords.), Los Derechos de la Naturaleza y la Naturaleza de sus Derechos, Ministerio de Justicia, Derechos Humanos y Cultos, Quito, 2011, pp 25-26; E.R. ZAFFARONI, "La Pachamama y el humano", in A. ACOSTA, E. MARTÍNEZ, (coords.), La naturaleza con derechos. De la filosofía a la política, Abya-Yala, Quito, 2011, pp. 133-136.

$20 \mathrm{Si}$ veda a tal proposito: E.R. ZAFFARONI, "La naturaleza como persona: de la Pachamama a la Gaia", in C. ESPINOSA GALLEGOS-ANDA, C. PÉREZ FERNÁNDEZ, (coords.), Los Derechos de la Naturaleza y la Naturaleza de sus Derechos, Ministerio de Justicia, Derechos Humanos y Cultos, Quito, 2011, p. 25.

${ }^{21} \mathrm{Si}$ veda a tal proposito: CORTE CONSTITUCIONAL DE COLOMBIA, Sentencia T-622/16, 2016. 
Per quanto concerne lo scenario delineato dai diritti bioculturali, è utile considerare che l'amministrazione e gestione dei territori delle comunità locali, che viene protetta e garantita dall'ordinamento giuridico, costituisce di per sé un primo ostacolo allo sfruttamento oltre ogni ragionevole criterio delle risorse naturali da parte di soggetti esterni mossi dalle sole logiche di profitto e dalla non curanza di alcuni di essi per i possibili ed eventuali danni.

In entrambe le tipologie di sistemi che proteggono la natura costituiscono una parte importante gli spazi offerti dagli ordinamenti giuridici per la partecipazione degli individui.

La partecipazione nei procedimenti tesi ad assumere determinate decisioni che riguardano i territori risulta fondamentale. Questa partecipazione per essere efficace deve essere previa, libera ed informata ${ }^{22}$. La partecipazione si sostanzia in veri e propri diritti procedimentali e coinvolge le persone nell'attività di protezione dell'ambiente ${ }^{23}$. Seppur essa si esplica in forme diverse in base a quanto previsto nei singoli ordinamenti giuridici, costituisce un complemento essenziale per la difesa della natura.

Per svolgere al meglio l'attività di partecipazione nei momenti in cui devono essere prese le decisioni, è necessario un adeguato accesso all'informazione. L'informazione si rende strumentale per la difesa della natura. I diritti all'informazione sono presidio della conoscenza completa di tutte le attività, la loro incidenza sul territorio e la possibilità che si verifichino danni ambientali. Inoltre, i diritti all'informazione comportano I'utilizzo di determinate forme per sensibilizzare le persone riguardo a determinati problemi ambientali24.

Dalla osservazione dei nuovi sistemi di protezione ambientale emerge una chiamata per gli individui a porre in essere comportamenti più consapevoli ed ecosostenibili, nell'ottica del rispetto dei diritti della natura o delle sue entità 0 in quella della coscienziosa gestione ed amministrazione dei territori. La figura umana che viene delineata è quella di un individuo consapevole dell'impatto ambientale delle proprie azioni, che non stia fermo nel menefreghismo, ma che si adoperi per arginare la deriva ambientale anche nelle proprie azioni quotidiane e che possa partecipare

\footnotetext{
22 Sull'informazione, sulla partecipazione e sulla «consulta previa, libre e informada» si veda: N. GREENE, G. MUÑOZ, Los Derechos de la Naturaleza, son mis Derechos. Manual para el tratamiento de conflictos socioambientales bajo el nuevo marco de derechos constitucionales, Plataforma de Acuerdos Socioambientales - PLASA, Colectivo Nacional por los Derechos de la Naturaleza, Programa de Pequeñas Donaciones del FMAM PPD/FMAM/PNUD, Quito, Ecuador, 2013, pp. 21-33.

${ }^{23}$ Si veda in tal senso: ivi, p. 30: «los derechos de participación, también conocidos como derechos procedimentales o conexos a los derechos ambientales sustantivos, en tanto ayudan a ejercer los derechos ambientales; responden a la necesidad de incorporar a personas y grupos en la protección ambiental».

${ }^{24} \mathrm{Si}$ veda in tal senso: ivi, p. 31: «el acceso a la información se convierte en un instrumento que permite un mayor conocimiento de una actividad que pueda afectar los recursos naturales, pero también en un instrumento que permite a los actores sociales generar mayor conocimiento y sensibilización sobre una problemática ambiental, así como decisiones más informadas al momento de tratar un conflicto socioambiental».
} 
ai procedimenti dove sono assunte le decisioni che riguardano il proprio territorio per contribuire alla difesa ambientale.

\section{CONCLUSIONI}

Negli ultimi anni si è compreso che l'impatto delle attività umane può compromettere il benessere del Pianeta. Alcuni Paesi stanno costruendo le proprie soluzioni per risolvere le problematiche ambientali basandosi sui valori culturali dei popoli che compongono i propri territori. I testi giuridici sono fortemente influenzati dalla cultura dei popoli. Essi diventano espressione della società, di cui ne veicolano i valori e la cultura. Il legame tra diritto e cultura è talmente forte che la cultura offre dapprima ispirazione per prospettive nuove nella costruzione del diritto, poi diventa valore fondante delle nuove costruzioni giuridiche e, infine, i testi giuridici divengono il mezzo con cui la cultura dei popoli che compongono il Paese si manifestano all'esterno. In concreto, cosa viene osservato nell'intreccio tra diritto e cultura? La cultura diviene il mezzo propulsivo di una nuova prospettiva, di un nuovo modo di costruire il diritto. Il mondo giuridico non è pensato più solo in funzione dell'essere umano, ma prende in considerazione un contesto più ampio dove ogni entità naturale ha una propria dignità e merita una particolare tutela. Il fenomeno che si osserva va sotto il nome di etnosviluppo giuridico ${ }^{25}$. Per etnosviluppo si intende il fenomeno in cui un popolo utilizza gli insegnamenti e la sapienza della propria tradizione per costruire il suo presente e futuro, proiettandovi le aspirazioni e i desideri insiti nella stessa società. Il diritto ambientale si arricchisce di contenuti in tal modo, progredisce, si perfeziona assecondando la visione già presente in seno alla società. Dinnanzi alla problematica ambientale, dove ogni Stato cerca soluzioni per porre freno alla deriva a cui la salute dell'ambiente si sta avviando, la risposta scelta da alcuni Paesi arriva dalla cultura e dalla tradizione delle comunità etniche e dai popoli indigeni che abitano il territorio.

Dall'intreccio tra diritto e cultura nascono due soluzioni interessanti in materia ambientale: i diritti bioculturali e i diritti delle entità naturali non umane.

I diritti bioculturali si fondano sul ruolo delle comunità locali o gruppi indigeni di amministrazione dei propri territori. Si tratta dei diritti tesi alla gestione tradizionale dei propri territori. Gli usi, le consuetudini, i rituali e le forme di autogoverno costituiscono l'esplicarsi del rapporto che le comunità locali hanno con l'ambiente circostante. $\grave{E}$, nello specifico, una relazione speciale che guarda con rispetto alla natura, la quale è gestita all'insegna della sostenibilità. Non vi sono logiche di mero profitto, ma le pratiche tramandate secondo la tradizione dei popoli che abitano il

$25 \mathrm{Si}$ veda a tal proposito: L. PERRA, "Etnodesarrollo jurídico y protección del medio ambiente", Veredas do Direito: Direito Ambiental e Desenvolvimento Sustentável, v. 16, n. 34, 2019, pp. 67-90. 
territorio hanno cura del perpetuarsi dei cicli vitali, del benessere della natura e delle sue entità.

I diritti delle entità naturali non umane costituiscono senza dubbio la novità più sorprendente nel mondo giuridico. La natura o le sue entità divengono soggetti o persone con veri e propri diritti. Il riconoscimento di questo carattere nel mondo giuridico segue una personificazione della natura o delle sue entità già presente nella cultura delle comunità etniche o dei popoli indigeni. Esse rivestono un'importanza notevole agli occhi di tali popoli che in un'ottica spesso sacrale impostano una vita in armonia con tutto l'ambiente che li circonda. La natura e le sue entità non sono antagoniste dell'essere umano, ma assieme a quest'ultimo formano un sistema più ampio dove gli equilibri e l'armonia contribuiscono al benessere generale.

L'essere umano, preso in considerazione nei nuovi sistemi di protezione ambientale dove emergono i diritti bioculturali o i diritti delle entità naturali non umane, è quello che tiene in considerazione l'ambiente nelle sue azioni quotidiane. Egli ha uno stile di vita ecosostenibile, con la natura convive in maniera armoniosa, ne rispetta i cicli vitali e non vede in essa un mero strumento attraverso il quale accrescere solo il proprio profitto economico. È la figura umana che predomina nella tradizione e nella cultura delle comunità etniche e dei popoli indigeni. L'uomo ha in sé il desiderio di proteggere la natura, il proprio ambiente, il proprio territorio, dove il benessere di ciascuna parte diviene quello generale.

\section{BIBLIOGRAFIA}

A. ACOSTA, "Los Derechos de la Naturaleza. Una lectura sobre el derecho a la existencia", in A. ACOSTA, E. MARTÍNEZ (coords.), La naturaleza con derechos. De la filosofía a la política, Abya-Yala, Quito, 2011, pp. 317-367.

J. ANSIÓN, "La interculturalidad como proyecto moderno", Páginas, v. 19, n. 129, 1994, pp. 9-16.

K.S. BAVIKATTE, T. BENNETT, "Community stewardship: the foundation of biocultural rights", Journal of Human Rights and the Environment, v. 6, n. 1, 2015, pp. 7-29. <https://doi.org/10.4337/jhre.2015.01.01>.

G. BONFIL BATALLA, "El etnodesarrollo: sus premisas jurídicas, políticas y de organización", in G. BONFIL BATALLA, M. IBARRA, S. VARESE, D. VERISSIMO, J. TUMIRI, et al. (F. Rojas Aravena, coord.), América Latina: Etnodesarrollo y Etnocidio, Ediciones FLACSO, San José, 1982, pp. 131145.

J. CRUET, La vie du droit et l'impuissance des lois, Ernest Flammarion, Paris, 1908.

E. CRUZ RODRÍGUEZ, "Derechos de la naturaleza, descolonización e interculturalidad. Acerca del caso ecuatoriano", Verba Iuris, n. 31, 2014, pp. 15-29. <https://doi.org/10.18041/0121-3474/verbaiuris.31.53>.

N. GREENE, G. MUÑOZ, Los Derechos de la Naturaleza, son mis Derechos. Manual para el tratamiento de conflictos socioambientales bajo el nuevo marco de derechos constitucionales, Plataforma de Acuerdos Socioambientales - PLASA, Colectivo Nacional por los Derechos de la Naturaleza, Programa de Pequeñas Donaciones del FMAM PPD/FMAM/PNUD, Quito, Ecuador, 2013. 
E. GUDYNAS, "Desarrollo, derechos de la naturaleza y Buen vivir despues de Montecristi," in G. WEBER (coord.), Debates sobre cooperación y modelos de desarrollo. Perspectivas desde la sociedad civil en el Ecuador, Centro de Investigaciones CIUDAD y Observatorio de la Cooperación al Desarrollo, Quito, 2011, p. 83-102.

P. HÄBERLE, Verfassungslehre als Kulturwissenschaft, Duncker \& Humblot, Berlin, 1998.

E.C. HSIAO, "Whanganui River Agreement - Indigenous Rights and Rights of Nature", Environmental Policy and Law, v. 42, n.6, 2012, pp. 371-375.

F. HUANACUNI MAMANI, Buen Vivir / Vivir Bien. Filosofía, políticas, estrategias y experiencias regionales andinas, Coordinadora Andina de Organizaciones Indígenas - CAOI, Lima, 2010.

E. MARTÍNEZ, A. ACOSTA, "Los Derechos de la Naturaleza como puerta de entrada a otro mundo posible", Revista Direito e Práxis, v. 8, n. 4, 2017, pp. 2927-2961. <https://doi.org/10.12957/dep.2017.31220>.

C. MASER, Earth in our care: ecology, economy, and sustainability, Rutgers University Press, New Brunswick, N.J., 2009.

M.J. MEDINA ESCALANTE, "Visión contemporánea acerca de los derechos humanos de los pueblos indios" in J.E.R. ORDÓÑEZ CIFUENTES (coord.), Pueblos indígenas y derechos étnicos: VII Jornadas Lascasianas, Universidad Nacional Autónoma de México, Instituto de Investigaciones Jurídicas, México, D. F., 1999, pp. 51-67.

L. PERRA, "Etnodesarrollo jurídico y protección del medio ambiente", Veredas do Direito: Direito Ambiental e Desenvolvimento Sustentável, v. 16, n. 34, 2019, pp. 67-90. <http://dx.doi.org/10.18623/rvd.v16i34.1385>.

L. PERRA, "Tradition Can Save the Future of Nature: Biocentric View of Law", in E.G. DOBBINS, M.L. PIGA, L. MANCA, (coords.), Environment, Social Justice, and the Media in the Age of the Anthropocene, Lexington Books, Lanham, MD, 2020, pp. 339-354.

A. PLA RODRÍGUEZ, "Derecho y cultura", Revista de la Facultad de Derecho, n. 10, 2005, pp. 85-94.

A. SALMOND, "Tears of Rangi: Water, power, and people in New Zealand", Hau: Journal of Ethnographic Theory, v. 4, n. 3, 2014, pp. 285-309. <https://doi.org/10.14318/hau4.3.017>.

J.M. VELÁZQUEZ-GUTIÉRREZ, "Constitucionalismo verde en Ecuador: Derechos de la Madre Tierra y Buen Vivir", Entramado, v. 10, n. 1, 2014, pp. 220238.

F. VIOLA, "Il futuro del diritto", Persona y Derecho, n. 79, 2018, pp. 9-36. $<$ https://doi.org/10.15581/011.79.9-36>.

E.R. ZAFFARONI, "La naturaleza como persona: de la Pachamama a la Gaia", in C. ESPINOSA GALLEGOS-ANDA, C. PÉREZ FERNÁNDEZ, (coords.), Los Derechos de la Naturaleza y la Naturaleza de sus Derechos, Ministerio de Justicia, Derechos Humanos y Cultos, Quito, 2011, p. 3-33.

E.R. ZAFFARONI, "La Pachamama y el humano", in A. ACOSTA, E. MARTÍNEZ, (coords.), La naturaleza con derechos. De la filosofía a la política, AbyaYala, Quito, 2011, pp. 25-137.

G. ZANETTI, "Diritti bioculturali: percorrendo la strada che separa diverse tradizioni", Diritto \& Questioni Pubbliche, v. 19, n. 1, 2019, p p. 269-272. 